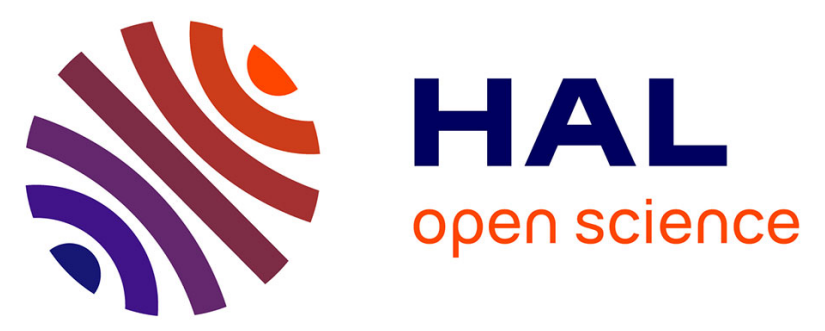

\title{
Reducing Data Acquisition for Fast Structured Illumination Microscopy using Compressed Sensing
}

William Meiniel, Piernicola Spinicelli, Elsa Angelini, Alexandra Fragola, Vincent Loriette, François Orieux, Eduardo Sepulveda, Jean-Christophe Olivo-Marin

\section{To cite this version:}

William Meiniel, Piernicola Spinicelli, Elsa Angelini, Alexandra Fragola, Vincent Loriette, et al.. Reducing Data Acquisition for Fast Structured Illumination Microscopy using Compressed Sensing. 14th International Symposium on Biomedical Imaging (ISBI 2017), Apr 2017, Melbourne, Australia. 10.1109/isbi.2017.7950461 . hal-01623739

\section{HAL Id: hal-01623739 \\ https://hal.science/hal-01623739}

Submitted on 5 Mar 2020

HAL is a multi-disciplinary open access archive for the deposit and dissemination of scientific research documents, whether they are published or not. The documents may come from teaching and research institutions in France or abroad, or from public or private research centers.
L'archive ouverte pluridisciplinaire HAL, est destinée au dépôt et à la diffusion de documents scientifiques de niveau recherche, publiés ou non, émanant des établissements d'enseignement et de recherche français ou étrangers, des laboratoires publics ou privés. 


\title{
REDUCING DATA ACQUISITION FOR FAST STRUCTURED ILLUMINATION MICROSCOPY USING COMPRESSED SENSING
}

\author{
William Meiniel ${ }^{1,2}$, Piernicola Spinicelli ${ }^{1}$, Elsa D. Angelini ${ }^{2,3}$, Alexandra Fragola ${ }^{4}$, \\ Vincent Loriette ${ }^{4}$, François Orieux ${ }^{5}$, Eduardo Sepulveda ${ }^{6}$, and Jean-Christophe Olivo-Marin ${ }^{1}$ \\ ${ }^{1}$ Institut Pasteur, BioImage Analysis Unit, CNRS UMR 3691, France. \\ ${ }^{2}$ LTCI, Telecom ParisTech, Universite Paris-Saclay, France. \\ ${ }^{3}$ ITMAT Data Science Group, NIHR Imperial BRC, Imperial College London, UK. \\ ${ }^{4}$ ESPCI ParisTech, LPEM, CNRS UMR 8213, France. \\ ${ }^{5}$ L2S, CNRS, Centrale Supelec, Universite Paris-Saclay, France. \\ ${ }^{6}$ LPNHE, IN2P3, CNRS UMR 7585, UPMC, France.
}

\begin{abstract}
In this work, we introduce an original strategy to apply the Compressed Sensing (CS) framework to a super-resolution Structured Illumination Microscopy (SIM) technique. We first define a framework for direct domain CS, that exploits the sparsity of fluorescence microscopy images in the Fourier domain. We then propose an application of this method to a fast 4-images SIM technique, which allows to reconstruct super-resolved fluorescence microscopy images using only $25 \%$ of the camera pixels for each acquisition.
\end{abstract}

Index Terms - Compressed Sensing, Structured Illumination Microscopy, Fluorescence microscopy.

\section{INTRODUCTION}

Over the last years, fluorescence microscopy has become a fundamental tool for modern experimental biology. Several techniques have been proposed to overcome the limitation related to the diffraction nature of the light [1. Among those, super-resolution far-field microscopy techniques include STED, PALM/STORM, and Structured Illumination Microscopy (SIM), introduced by Gustaffson in 2000 [2] SIM is one of the less invasive super-resolution imaging technique for biological samples. It can be seen as an extension of widefield microscopy where the biological sample is illuminated with a series of sinusoidal patterns of high spatial frequencies. Such illuminations inject high frequencies inside the optical transfer function of the microscope objective through the appearance of Moiré fringes as a result of interferences between the illumination patterns and the biological sample details.

Although SIM has already demonstrated its capability to achieve fluorescence imaging with enhanced spatial resolution, it has not been widely used yet for biological application because of the difficulty to reconstruct the images. Fast dynamic studies of living samples are still limited by the number of required raw image acquisitions (9 images at least) to obtain one high-resolution image. Moreover, with the rapid development of new CMOS-based scientific cameras with higher pixel density, both fast transfer and computation of huge amount of data could become a bottleneck for the diffusion of this new super-resolution strategies.
In this work, we propose an original method, based on the Compressed Sensing (CS) theory, to overcome these drawbacks by reducing the amount of acquired data needed to reconstruct a super-resolved SIM image by a factor up to 4, without loosing in resolution or signal-to-noise ratio.

\section{DIRECT DOMAIN COMPRESSED SENSING}

\subsection{Compressed Sensing Theory}

Compressed Sensing (CS) is a mathematical theory originally developed in the works of Candès [3] and Donoho [4] in 2006. This theory states that a sparse signal can be recovered from a drastically low amount of randomly sampled data. In the case of image acquisition, we want to reconstruct the true image $x \in \mathbb{R}^{N}$ from a given observation $y=\Phi x \in \mathbb{R}^{M}$ (with $M<<$ $N)$, where $\Phi \in \mathbb{R}^{M \times N}$ denotes the measurement operator. The image $x$ has a sparse representation with respect to a known dictionary $\Psi \in \mathbb{R}^{N \times L}$. It means that there is a set of coefficients $s \in \mathbb{R}^{L}$ with only $S$ non-zero values $(S<<N)$, such that $x=\Psi s$. The CS theory shows that, under some constraints [3] it is possible to recover an estimator $\hat{x}_{C S}$ of the true image $x$ from the observation $y$ by solving the following optimization problem:

$$
\hat{x}_{C S}=\underset{x \in \mathbb{R}^{N}}{\arg \min }\left\|\Psi^{\dagger} x\right\|_{1} \text { such that } \Phi x=y
$$

where $\Psi^{\dagger}$ is the pseudo inverse of the operator $\Psi$.

\subsection{Fourier-domain sparsity}

The notion of sparsity is central in the CS theory, but natural or biological images are seldom sparse. On the other hand, in the context of microscopy, all images share one common property: they are the results of a convolution between the true illuminated scene and the point spread function (PSF) of the microscope. This leads to a loss of resolution, which we can model as a low-pass filter in the Fourier domain. It can be modeled via setting to zero most of the Fourier coefficients of the image, which corresponds to a sparse Fourier transform, with a degree of sparsity directly proportional to the spatial support of the PSF of the system.

Most CS applications consider random sampling in the Fourier domain to reconstruct an image that is sparse (or 
more realistically compressible) in the spatial domain (or some transform domain). We propose in this work to follow the opposite approach, by enforcing sparsity in Fourier domain and sampling sparsely in spatial domain.

Our results demonstrate that it is possible to recover a SIM image $x$ from a limited collection $y$ of camera pixels, with a controlled reconstruction error (see [5]).

We define the operators $\Phi$ and $\Psi$ as follows. First, the sensing operator $\Phi \in\{0,1\}^{M \times N}$ is a direct-domain selection matrix, where $M$ is the number of pixels selected among the $N$ pixels of the camera. The sampling rate is defined as $\tau=$ $\frac{M}{N} \in[0,1]$. The sparsifying transform is simply defined as the Fourier transform operator $\mathcal{F}$.

The CS optimization problem (1) is written as:

$$
\hat{x}_{C S}=\underset{x \in \mathbb{R}^{N}}{\arg \min }\|\mathcal{F}(x)\|_{1} \text { such that } \Phi x=y
$$

which we solve using the NESTA algorithm [6].

\section{FAST STRUCTURED ILLUMINATION MICROSCOPY}

SIM is based on the illumination of a given biological sample with a series of structured patterns, to observe the resulting interferences through a microscope, and reconstruct a superresolved image from these observations. In the context of linear SIM, the illuminations patterns are sinusoidal modulations, that can be written, for each pixel location $(p, q)$ as:

$$
I(p, q)=I_{0}\left(1+\alpha \cos \left(2 \pi\left(k_{p} p+k_{q} q+\varphi\right)\right)\right)
$$

where $I_{0}$ is the mean intensity of the illumination, $\alpha$ is the modulation depth, $\left(k_{p}, k_{q}\right)$ encodes its orientation and frequency, and $\varphi$ its phase.

In standard SIM implementation [2], $K=9$ illuminated observations are made to reconstruct a single SIM image. In [7, an alternative approach to the standard SIM image reconstruction was proposed, based on a Bayesian inverse problem formulation strategy that only requires $K=4$ raw images to generate a super-resolved image. In the following, we will refer to this technique as SIM4i.

\subsection{SIM reconstruction}

Mathematically, the acquisition of each raw image $g_{k} \in \mathbb{R}^{N}$, $k=[1,2, ., K]$ can be modeled as follows:

$$
g_{k}=H I_{k} f+n_{k}
$$

where $f \in \mathbb{R}^{N}$ is the high-resolution image to recover, $H$ represents the PSF of the microscope, $I_{k}$ is the illumination pattern of the observation $k$, and $n_{k}$ is the noise term corrupting the observation $k$. The illumination pattern does not change the low-pass effect of the PSF, but the frequency of the modulation needs to fall within the support of the PSF spectrum.

Then, if we collect the $K$ raw images in one vector $g=$ $\left[g_{1}, \ldots, g_{K}\right]$, the general model can be written:

$$
g=\tilde{H} I R f+n
$$

where $\tilde{H}$ and $I$ are block diagonal matrices containing respectively $H$ and $I_{k}$ in each block, $R$ is a replication matrix and $n$ is the overall noise term.
The SIM4i technique solves an inverse problem that is written [7]:

$$
\hat{f}_{S I M}=\underset{f \in \mathbb{R}^{N}}{\arg \min }\|g-\tilde{H} I R f\|_{2}^{2}+\lambda\|D f\|_{2}^{2}
$$

where $\lambda$ is a hyperparameter determined through Bayesian estimation, and $D f$ is the Hessian of the image $f$.

\subsection{CS-SIM}

In this work, we propose to acquire only a subset of $M$ randomly chosen camera pixels of each raw observation. Using the notations defined above, the acquisition model (3) becomes:

$$
g_{k}=\Phi\left(H I_{k} f+n_{k}\right)
$$

Now, we solve the optimization problem 2, to reconstruct an estimator $\hat{g}_{k, C S}$ from the raw observation $g_{k}$. Finally, collecting the $K$ estimators $\hat{g}_{k, C S}$ into a vector $\hat{g}_{C S}$, we can apply the SIM4 $i$ inverse problem (5), and write the CS-SIM technique as:

$$
\hat{f}_{C S-S I M}=\underset{f \in \mathbb{R}^{N}}{\arg \min }\left\|\hat{g}_{C S}-\tilde{H} I R f\right\|_{2}^{2}+\lambda\|D f\|_{2}^{2}
$$

\section{EXPERIMENTAL RESULTS}

\subsection{Dataset}

We tested the CS-SIM algorithm on two images. The first one is a synthetic target image, designed for testing superresolution methods [8]. We display in Fig 1 2 only part of the original image (matrix size $=400 \times 400$ pixels, pixel size $=25 \mathrm{~nm}$ ) for better clarity. The second image is a fluorescence microscopy image of Hela cells (matrix size $=400 \times 400$ pixels, pixel size $=100 \mathrm{~nm}$ ), acquired in our unit at Pasteur Institute. Both images serve as ground-truth high-resolution (HR), which we degrade, to simulate widefield observations, with a smoothing PSF modeled as an Airy disc with central disc diameter of 10 pixels.

We simulated CS-SIM acquisition on both images. As in [7], four modulation grids were used at angles $\left[0,0, \frac{\pi}{3}, \frac{2 \pi}{3}\right]$, phases $\varphi=\left[0, \frac{2 \pi}{3}, 0,0\right]$, and frequency adapted to the size of the PSF. The noise term is chosen to be white Gaussian with standard deviation $\sigma=1$. Finally, the sensing operator $\Phi$ is a uniform random selection of either $\tau=25 \%$ or $\tau=50 \%$ of the total number of pixels in each observation (i.e. camera captors).

\subsection{Qualitative results}

The two images described in Sec 4.1 are used to evaluate the quality of our CS-reconstruction and CS-SIM techniques. We display in Fig 1.2 visual illustrations for some CSreconstruction and CS-SIM results, with $\tau=25 \%$ (that corresponds to our goal for fast-SIM imaging) and $\tau=50 \%$.

\subsubsection{CS reconstructions}

On Fig 1 , we display the HR images and their widefield observation, along with the Fourier Transforms. This visualization 
confirms the validity of Fourier-domain sparsity, with the majority of the high-frequency coefficients (outside the central disc) encoding the noise.

The third row displays, for each HR image $x$, the original subsampled data $y$ with a sampling rate $\tau=25 \%$, as well as its Fourier Transform $\mathcal{F}(y)$. We also display the CSreconstruction results of the widefield image for $\tau=25 \%$, that is our target sampling rate, and $\tau=50 \%$, which shows some visual improvement compared to $\tau=25 \%$.

Comparison of the Fourier transform of the CS reconstruction and the original widefield image shows artifacts along the central vertical axis, which falls into the standard level of noise. Hence, these artifacts are negligible on the direct domain images.

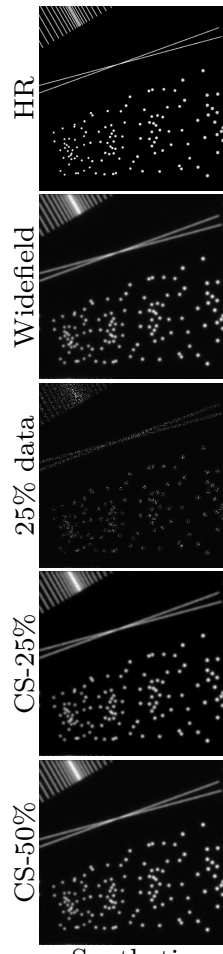

Synthetic
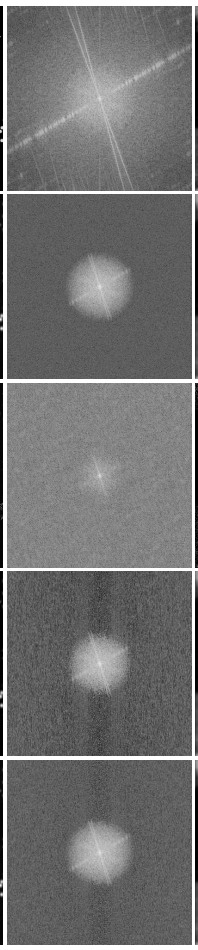

F.T.

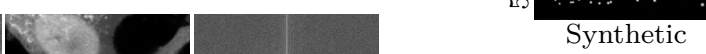

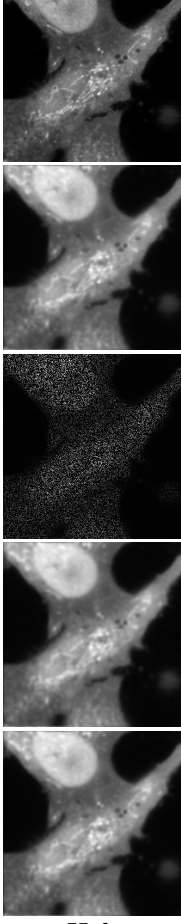

Hela
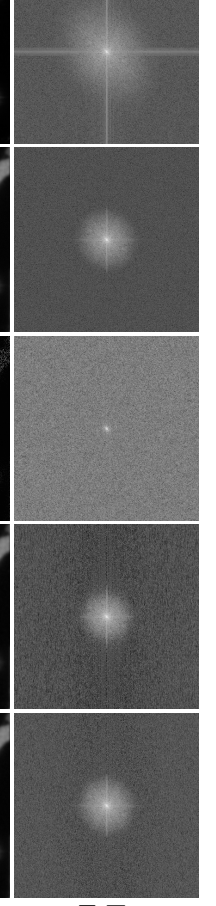

F.T.

Fig. 1: High-resolution (HR), widefield observations and CS-reconstructions (with $\tau=25 \%$ or $50 \%$ ) of the widefield data on the Synthetic and Hela images, along with their Fourier Transforms (F.T.), shown in log-values for visualization. Third row represents the spatial random sampling collected on the camera, along with its Fourier Transform.

\subsection{2. $C S-S I M$}

After applying CS-reconstruction on each of the $K=4$ modulated observations, we display in Fig 23 the CS-SIM reconstruction results for both test images, with $\tau=25 \%$ and $\tau=50 \%$. We clearly see the super-resolution effect of SIM, increasing the spatial extent of the Fourier spectrum of the reconstructed image. The support size is the same for both $\tau=25 \%$ and $\tau=50 \%$, enabling to maintain the resolution gain of a factor 2 .

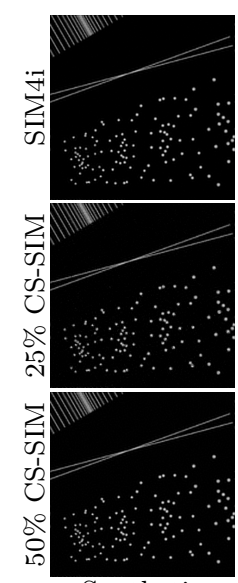

Fig. 2: SIM $4 i$ and CS-SIM reconstructions ( $\tau=25 \%$ or $50 \%)$ of the Synthetic and Hela images, along with their Fourier Transforms (F.T.).

We provide in Fig 3 zoomed views of both series of results, enabling visual assessment of the quality of the reconstruction at the pixel level. We can see that resolution is already greatly improved with $\tau=25 \%$, and almost optimal (with respect to the original SIM $4 i$ image) for $\tau=50 \%$. A segment was selected in both image sets and intensity profiles are displayed, to illustrate contrast improvement in an intermediate range between widefield observation and reference HR image.

The two zoomed areas were chosen to illustrate the power of resolution improvement in separating adjacent structures such as two high-contrast spots or three low-contrast linear shapes. The HR images show separate structures, while the widefield images merge them and distort their shapes. We see that the original SIM $4 i$ technique and CS-SIM reconstructions are able to recover separate objects, with better estimates of the intensity levels in low-contrast areas.

\subsection{Quantitative results}

To quantify the visual quality of the reconstructed images, three metrics are used: Mean Square Error (MSE), Structural Similarity Index Measure (SSIM) 9], and normalized Fourier Spectrum Analysis (FSA) [10]. The FSA computes the average energy along concentric circles of radius inversely proportional to resolution (see Fig 4). Both MSE and SSIM measurements use a reference image for comparison, which is supposed to be the ideal image. Accordingly, we used as our reference the widefield images to evaluate CS-reconstructions, and the HR images to evaluate CS-SIM reconstructions. All measures, tested versus the sampling rate value $\tau$, are reported in Fig 4

The normalized FSA measures show that our CS-SIM method recovers the Fourier information as efficiently as the original SIM4 $i$ method, with $\tau$ as low as $25 \%$.

For both MSE and SSIM, CS-reconstruction measures improve quite fast with the sampling rate. Even though the error is never exactly zero, we notice that the CS-SIM reconstruction provides strong improvement in this direction. Indeed, in terms of MSE, the CS-SIM reconstruction is al- 


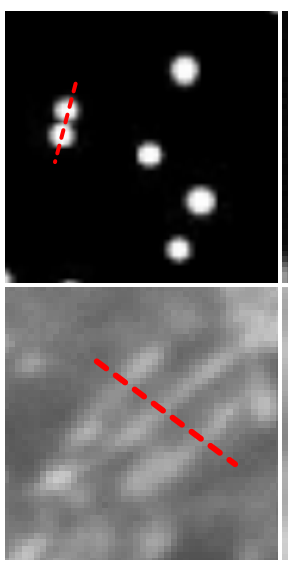

HR image

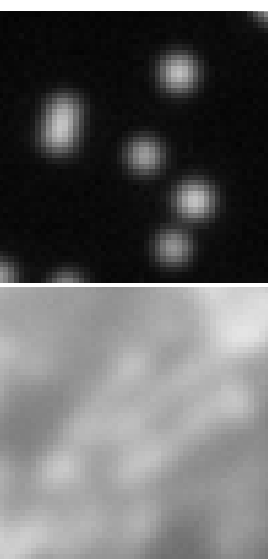

Widefield

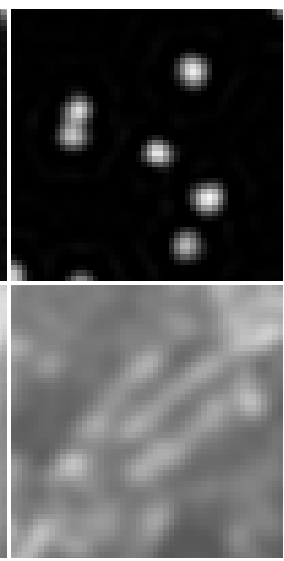

SIM4i
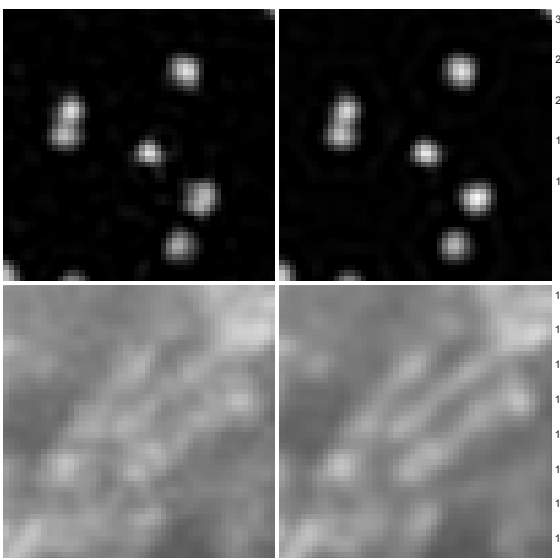

$25 \%$ CS-SIM

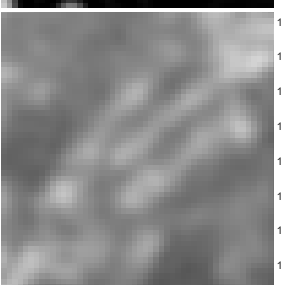

$50 \%$ CS-SIM

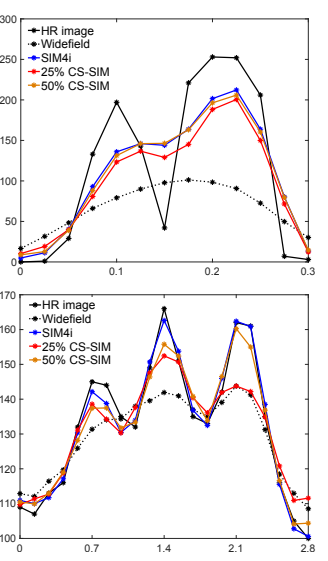

Profiles

Fig. 3: Magnified view (patch size $=50 \times 50$ pixels) of the CS-SIM reconstructions from Fig.2 on structural details for the Synthetic and Hela images. The last column displays intensity profile values along the red dotted segments shown on the HR images. Unit of $x$ axis is in $\mu m$.

ready almost exact for a sampling rate of $40 \%$. The SSIM keeps improving as the sampling rate grows, which visually corresponds to elimination of small artifacts around the edges of tiny objects inside the images.

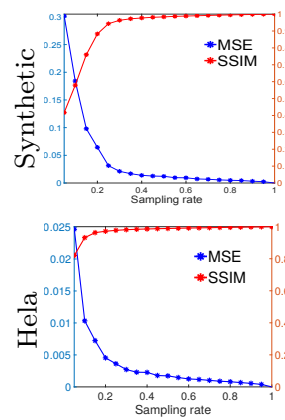

CS-reconstruction
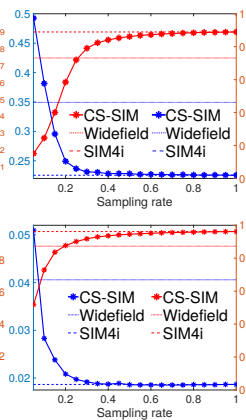

CS-SIM

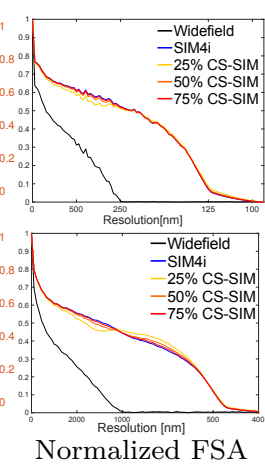

Normalized FSA
Fig. 4: Quantitative evaluations of the CS reconstructions versus widefield images and the CS-SIM reconstructions versus HR images. For each technique, we report MSE (in blue) and SSIM (in red) measurements with respect to the sampling rate used by the CS. On the third column, we report the normalized FSA measures for the different reconstructions.

\section{CONCLUSION}

In this work, we have explored a Compressed Sensing based approach for Structured Illumination Microscopy imaging, and provided a new framework for fast super-resolution microscopy. Via simulations of SIM acquisitions we have shown that CS-SIM allows SIM reconstruction of equal quality but with only $25 \%$ of the camera pixels sampled and stored during acquisition of each of the modulated images. This paves the way toward new opportunities such as live-SIM. In the near future, we will apply this method to video sequences, and as soon as scientific cameras with random selection of pixels are available, we will implement our method on a real experimental set-up.

\section{ACKNOWLEDGMENTS}

The authors would like to thank Maria Manich from Institut Pasteur for the acquisition of the Hela cells images. This work was partially funded through grants from the Agence Nationale de la Recherche (ANR-10-INSB-04-06 France Bioimaging, ANR-10-LABEX62-IBEID), Carnot Pasteur Maladies Infectieuse (ANR 11-CARN 01701), and through a PhD fellowship from the Ecole Normale Supérieure de Cachan.

\section{REFERENCES}

[1] L. Schermelleh, R. Heintzmann, and H. Leonhardt, "A guide to super-resolution fluorescence microscopy," The Journal of Cell Biology, vol. 190, no. 2, pp. 165-175, 2010.

[2] M.G.L. Gustafsson, "Surpassing the lateral resolution limit by a factor of two using structured illumination microscopy," Journal of Microscopy, vol. 198, no. 2, pp. 82-87, 2000.

[3] E.J. Candès, J. Romberg, and T. Tao, "Robust uncertainty principles: Exact signal reconstruction from highly incomplete frequency information," Transactions on Information Theory, vol. 52, no. 2, pp. 489-509, 2006.

[4] D.L. Donoho, "Compressed sensing," Transactions on Information Theory, vol. 52, no. 4, pp. 1289-1306, 2006.

[5] E. Candès, "The restricted isometry property and its implications for compressed sensing," Comptes Rendus Mathematique, vol. 346, no. 9 - 10, pp. 589 - 592, 2008.

[6] S. Becker, J. Bobin, and E. Candès, "Nesta: A fast and accurate first-order method for sparse recovery," Journal on Imaging Sciences, vol. 4, no. 1, pp. 1-39, 2011.

[7] F. Orieux, E. Sepulveda, V. Loriette, B. Dubertret, and J-C. Olivo-Marin, "Bayesian estimation for optimized structured illumination microscopy," Transactions on Image Processing, vol. 21, no. 2, pp. 601-614, 2012.

[8] K. Wicker, "Non-iterative determination of pattern phase in structured illumination microscopy using auto-correlations in fourier space," Optics Express, vol. 21, no. 21, pp. 24692-24701, 2013.

[9] Z. Wang and A.C. Bovik, "Mean squared error: Love it or leave it? a new look at signal fidelity measures," Signal Processing Magazine, vol. 26, no. 1, pp. 98-117, 2009.

[10] J. Demmerle, E. Wegel, L. Schermelleh, and I.M. Dobbie, "Assessing resolution in super-resolution imaging," Methods, vol. 88 , pp. 3-10, 2015. 